\title{
Gestión De Una Red Eléctrica De Distribución Ante Desastres A Partir De Recursos Energéticos Distribuidos
}

\author{
Yenni Marcela Espinosa Rico , Sebastián Camilo Becerra Cortes, Luis Alejandro Arias \\ Barragán
}

Universidad Autónoma De Colombia

Recibido: 15/04/2021 Revisado: 21/05/2021 Aceptado: 26/06/2021 Publicado: 28/07/2021

\section{Resumen}

En este artículo se ha llevado a cabo una revisión de literatura científica con respecto al tema de resiliencia estableciéndose una línea de tiempo desde el año 2000 hasta 2020 abordando diferentes temas de los recursos energéticos distribuidos (DER) y su influencia en la resiliencia energética. A partir de dicha revisión, se ha realizado un análisis estadístico y una proyección probabilística de las tendencias de desarrollo a futuro sobre el tema.

Este trabajo de investigación enfoca en el desarrollo de una estrategia para fortalecer la gestión una red eléctrica de distribución de manera dinámica que se ajuste a los requerimientos funcionales como calidad de la energía eléctrica suministrada y la máxima cobertura posible a cargas de alta prioridad como hospitales y centros de atención, en periodos durante y después de la ocurrencia de un desastre natural como un movimiento telúrico. Con el fin de apoyar y afrontar la situación de un desastre, se propone utilizar recursos energéticos distribuidos apoyados en elementos de Generación Distribuida (GD), sistemas de almacenamiento y recursos de Respuesta de la Demanda generada que son gestionados mediante sistemas de control automatizado.

\section{Palabras claves}

Resiliencia, recursos energéticos distribuidos, evolución de la resiliencia en sistemas eléctricos, teoría de grafos; seccionamiento de red 


\begin{abstract}
In this article, a scientific literature review has been carried out regarding the issue of resilience, establishing a timeline from 2000 to 2020 addressing different issues of distributed energy resources (DER) and their influence on energy resilience. From this review, a statistical analysis and a probabilistic projection of future development trends on the subject have been carried out.
\end{abstract}

This research work focuses on the development of a strategy to strengthen the management of an electrical distribution network in a dynamic way that adjusts to functional requirements such as quality of the electrical energy supplied and the maximum possible coverage to high priority loads such as hospitals and centers of attention, in periods during and after the occurrence of a natural disaster such as an earthquake. In order to support and face the situation of a disaster, it is proposed to use distributed energy resources supported by Distributed Generation (DG) elements, storage systems and generated Demand Response resources that are managed by automated control systems.

\title{
Keywords
}

Resilience, distributed energy resources, evolution of resilience in electrical systems, graph theory; network sectioning 


\section{Introducción}

En el presente escrito, se ha realizado una recopilación de trabajos sobre resiliencia en sistemas energéticos ante eventos disruptivos como terremotos u otros desastres naturales, durante los años de 2000 a 2020. De igual forma, se revisa el uso que se le ha dado a los DER ante estos eventos y la evolución en procesos de integración dentro de la red eléctrica.

El análisis de tendencias de desarrollo en materia de inclusión de DER para apoyar en el mantenimiento del suministro energético en la red ante desastres naturales muestra que se da una prelación particular a las energías renovables ubicadas en usuarios puntuales a nivel residencial en viviendas multifamiliares y comerciales que puedan ser afectadas por un evento catastrófico de la red.

El presente texto se encuentra estructurado de la siguiente forma: Inicialmente, se tiene la introducción a la temática sobre la resiliencia en sistemas energéticos y la participación de los DER como soporte de suministro de energía ante contingencias; posteriormente se muestra la línea de tiempo de artículos revisados y la clasificación de acuerdo a categorías que autores han determinado como relevantes, se tiene el análisis estadísticos; se realiza una discusión de resultados obtenidos y algunas propuestas de desarrollo a futuro para sistemas resilientes que se poyen en DER; Finalmente se tienen las conclusiones del trabajo realizado.

\section{Situación actual de la resiliencia de sistemas eléctricos y redes eléctricas}


Las redes energéticas actuales dentro de sus características principales están garantizar una cobertura amplia, continuidad en el servicio y niveles admisibles de calidad en la tensión entregada a los clientes(Landau, 1937), deben ser resiliente ante fallos. El concepto de resiliencia surgió por primera vez por (holling, 1793) cuando la definió como "una medida de persistencia de los sistemas y de su capacidad para absorber cambios y perturbaciones y aún mantener las mismas relaciones entre poblaciones o variables de estado", más adelante (Gunderson,1995) agrego el concepto de capacidad de almacenamiento, (Walker,2002) amplio aún más la definición agregando el concepto de autocorregirse durante las perturbaciones, (Kendra,2003) introdujo el concepto de "recuperarse de una perturbación". (Woods, 2017) define la resiliencia como la capacidad de ser inmune para futuros eventos similares, y más recientemente (Hollnagel, 2017) la define como la capacidad de un sistema para "ajustar su funcionamiento antes o durante los eventos (cambios), perturbaciones y oportunidades) y mantener las operaciones requeridas en condiciones esperadas como inesperadas".

En el contexto energético, se define la resiliencia como "la capacidad de recuperación de un sistema de energía para tolerar perturbaciones y continuar brindando servicio de energía asequibles a los consumidores" y el sistema resiliente de energía puede recuperarse rápidamente de daños y proporcionar medios alternativos para satisfacer de energía a las necesidades de servicios en circunstancias extremas (Chaudry, 2011)(Bajwa et al., 2019).

La figura 1, describe el concepto de curva de resiliencia basados en las principales características, teniendo en cuenta al momento de presentarse un evento extremo. Para (Panteli \& Mancarella, 2015) el primer estado es el estado resiliente, que representa el sistema de potencia antes de que ocurra un evento, las principales características de este estado son robustez/resistencia lo que implica que el sistema debe ser lo suficientemente confiable y robusto para soportar un daño inicial. Posteriormente, sigue el estado "degradado posterior al evento" que se da después de someter el sistema a una perturbación, se caracteriza por la flexibilidad operativa para adaptarse a las 
condiciones cambiantes (ingenio, redundancia y auto-organización), ayudando a apaciguar el evento. A continuación, el sistema entra en el "estado restaurador", donde el sistema debe exhibir una respuesta rápida para asegurar una máxima recuperación en cada estado resiliente tan rápido como sea posible. Finalmente, el sistema energético entra en el "estado post-restauración", donde el nivel de la resiliencia puede o no ser tan alto como el estado pre-evento dependiendo de la robustez y resistencia posterior al evento. Esto significar que el sistema puede o no haber recuperado su capacidad máxima de operación.

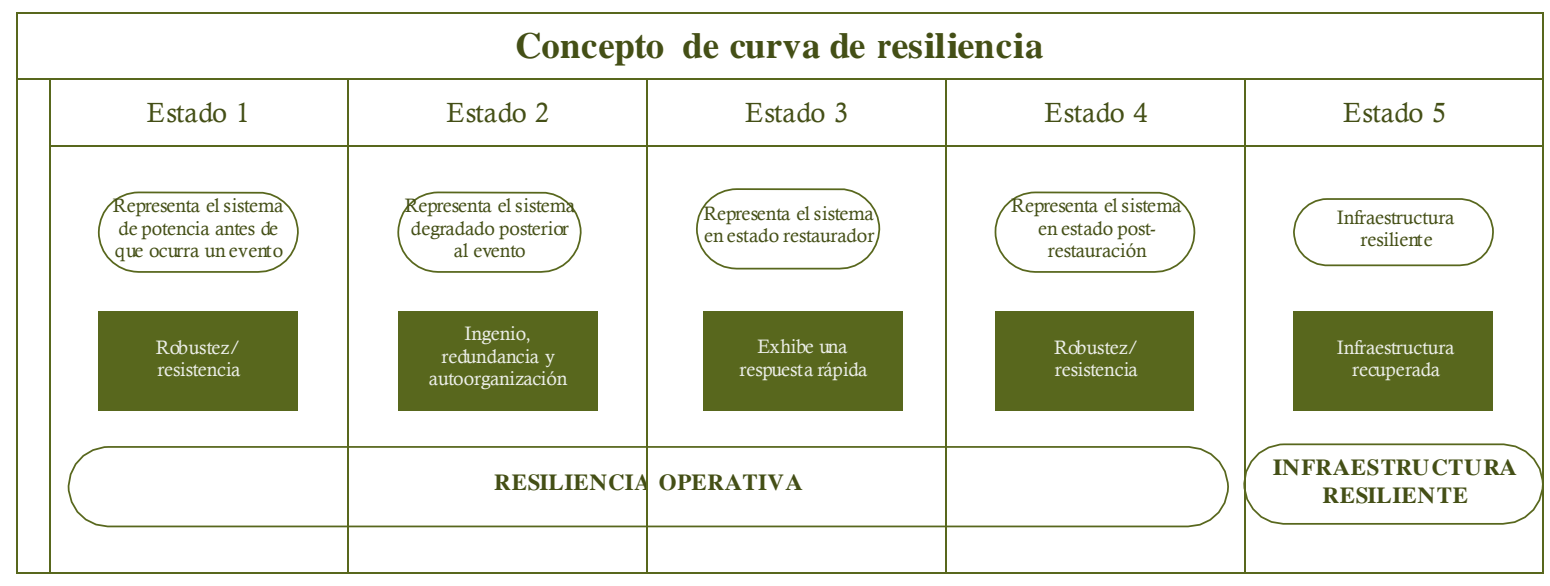

Figura 1. Concepto de curva resiliente asociada a un evento.

La figura 2, muestra la tendencia entre los diferentes periodos de tiempo; la mayoría de los estudios realizados han sido sobre recursos energéticos distribuidos en periodos comprendido entre 2004 y 2008 fue de 71,4\%, entre 2009 y 2012 fue de 63,63\%, entre 2013 -2016 es de 61,9\% y finalmente 2017 -2020 es 46,1\% mientras que en términos de resiliencia y sistemas de almacenamiento fueron en menor cantidad, posteriormente por sistemas de gestión de redes ante desastres que ha ido aumentando su intervención y en el último periodo equivale a un 30,8\%. 


\section{Estudios a través de los años}

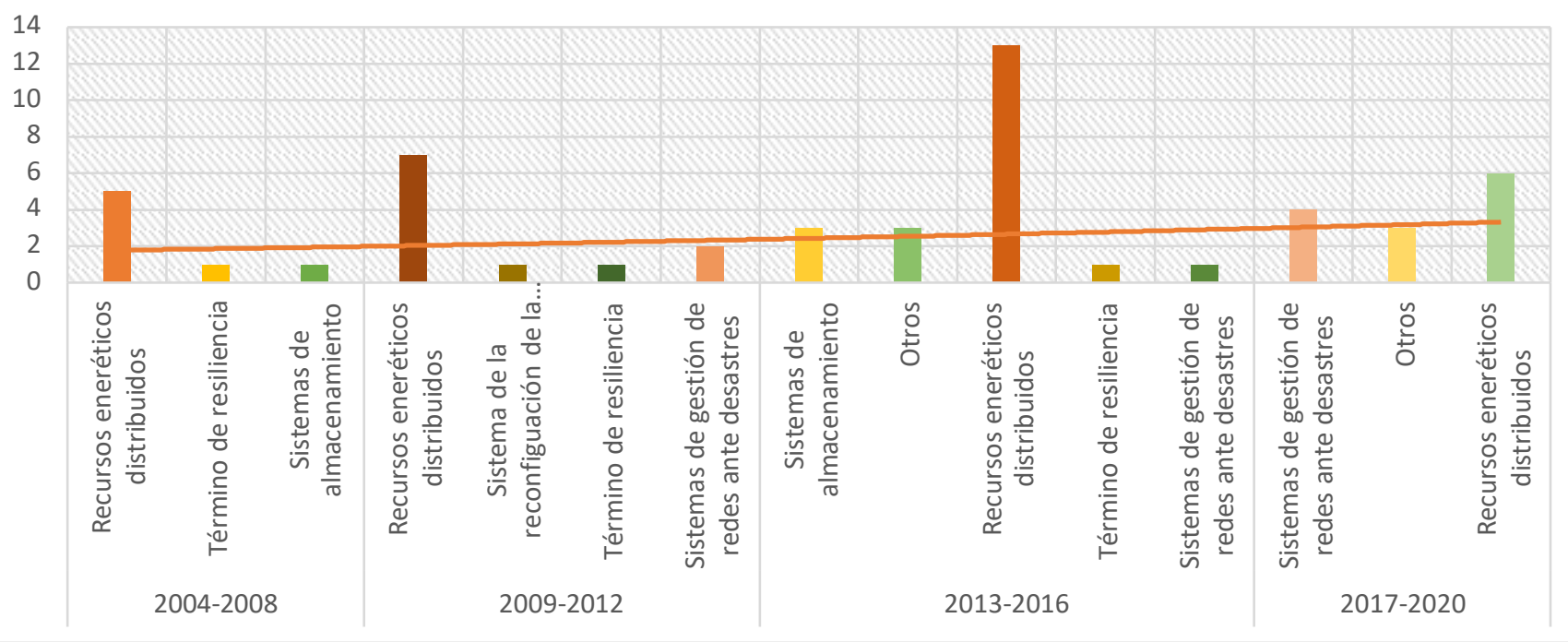

Figura 2. Estudios realizados a través de los años

\section{Teoría de grafos y su aplicación}

Un grafo $\mathrm{G}$ consiste de una colección de vértices $\mathrm{V}$ (nodos) y una colección de líneas $\mathrm{E}$ (aristas). Como se ve en la ecuación (ec1).

$$
\mathrm{G}=(\mathrm{V}, \mathrm{E}) \quad(\mathrm{ec} 1)
$$

Comparándolo a una red de distribución de electricidad, el conjunto de nodos $\mathrm{V}$ incluye subestaciones, transformadores de distribución, interruptores, barras de distribución y ubicaciones de los consumidores. El conjunto de líneas E, representa las conexiones físicas entre los nodos a través de cables subterráneos y segmentos de líneas aéreas de distribución.

Matriz de adyacencia: La conectividad entre nodos en un grafo está representada por una Matriz de adyacencia 'A'. Dado que la red eléctrica puede considerarse como un grafo no dirigido, la 
matriz de adyacencia de una red eléctrica se convierte en una matriz $\mathrm{NxN}$ simétrica. El elemento Aij se convierte en 1 si existe un enlace entre los nodos i y j, de lo contrario, Aij es igual a 0.

Matriz de Grado: El Grado de un nodo i en un gráfico que se denota como ki, es el número de líneas incidentes en ese nodo y se obtiene utilizando la matriz de adyacencia. Los valores obtenidos para el Grado de cada nodo se utilizan para construir la Matriz de Grado "K", que es una Matriz diagonal NxN.

La matriz laplaciana 'L' es útil para obtener las propiedades gráficas de las redes eléctricas y se denota con la letra "L". Es representada en la ecuación 2 (ec2).

$\mathrm{L}=\mathrm{K}-\mathrm{A} \quad(\mathrm{ec} 2)$

En la figura 3, se ilustra el algoritmo inicial de seccionamiento fundamentado en la teoría de grafos. 


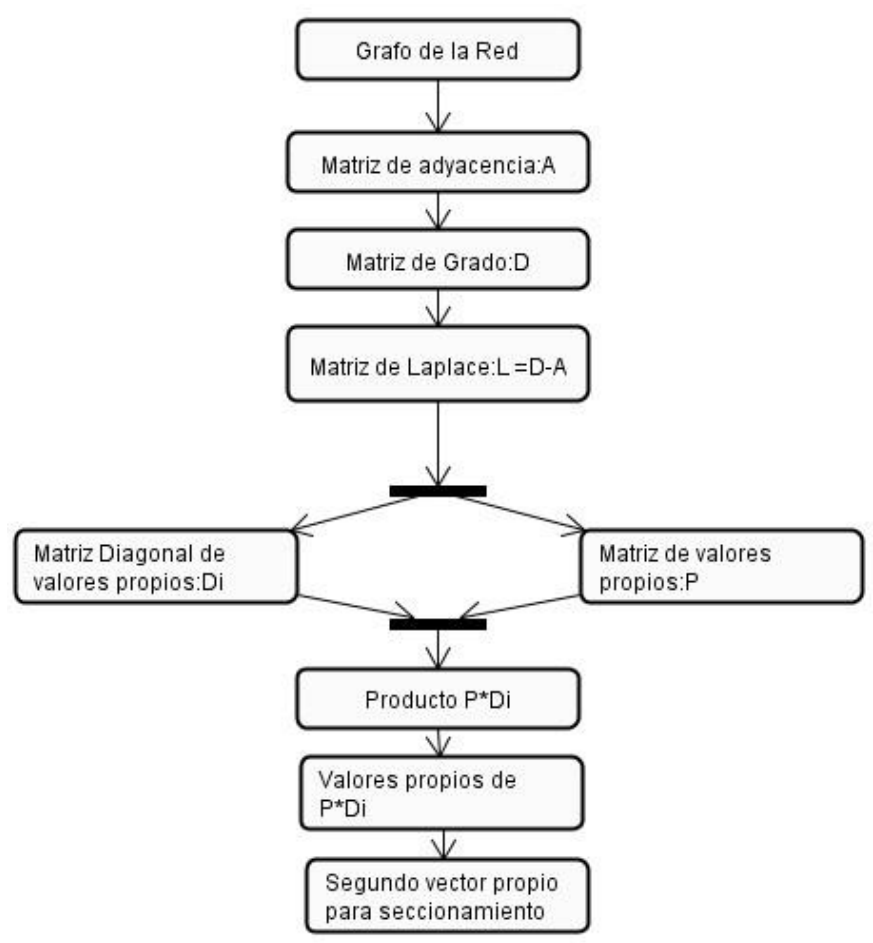

Figura 3. Algoritmo Inicial de grafos

\section{Planteamiento del Problema y Justificación}

En el estado actual de la red a nivel de distribución se observa una dispersión de los recursos energéticos distribuidos (DER) con una baja apropiación de los mismos en la gestión de los recursos energéticos por parte del Operador de Red a la par con la inexistencia de mecanismos suficientes para el restablecimiento del suministro energético en el momento de ocurrencia de desastres naturales que afecten gravemente la infraestructura eléctrica existente. En la literatura científica se evidencia que existe una estructura centralizada y vertical de la administración y de las operaciones en la red de distribución eléctrica por parte de un Operador de Red, resultando en un pobre desempeño de la red en general (Niknam, T. , Ranjbar A.M., Shirani A.R., 2005) (Bouffard \& Kirschen, 2008) (Levin \& Thomas, 2016). 
Por otra parte, la provisión de los servicios auxiliares, tales como la cobertura de picos de demanda, la confiabilidad en el suministro eléctrico y compensación de reactivos por parte de las empresas de distribución y/o el Operador de Red se realiza mediante inversiones propias en infraestructura o bien subcontratando con otras empresas por regla general de tipo privado (CREG (Comisión de Regulación de Energía y Gas), 2010) (Quintero, 2013). De acuerdo a lo anteriormente expuesto, existe una desarticulación entre los problemas de desempeño de la red a nivel de distribución y de provisión de servicios auxiliares; y los recursos energéticos distribuidos que pueden suplir dichas necesidades, especialmente ante la ocurrencia de desastres.

La propuesta de Investigación plantea estrategias de gestión de DER apoyadas en una plataforma de acceso que se tiene en espacio de Computación en la nube, donde se realiza el almacenamiento de datos de la red, la administración de los recursos energéticos, el manejo de datos estocásticos de los DER, para el caso concreto RD, GD y sistemas de almacenamiento con el fin de atender situaciones de emergencia de desabastecimiento en el sistema energético cuando ocurre un desastre natural.

\section{Justificación}

En el contexto actual de desarrollo de los sistemas energéticos se identifican tendencias como: el mejoramiento de la operatividad del sistema, la integración de los recursos energéticos distribuidos, la inclusión de los vehículos eléctricos en las redes de distribución, la expansión de la Respuesta a la Demanda y la evolución de los mercados energéticos (Hammons, 2008) (Kassakian et al., 2011) (International Energy Agency, 2013) . Referente a la Integración de DER en las redes de distribución en la literatura científica se ha propuesto el desarrollo de herramientas como modelos, algoritmos y estrategias, que responden a funciones objetivo variables en el tiempo y con criterios de evaluación técnico, económicos, social, normativo y medio-ambiental (Sismotto \& Hage, 2005), (Sadeghi et al., 2012) (Di Somma et al., 2015). 
De igual forma se evidencia la necesidad de diseñar estrategias y mecanismos que permiten mejorar la resiliencia de los sistemas energéticos ante situaciones de desastres naturales que se apoyen en los DER que los usuarios de las redes poseen y que pueden poner al servicio de un operador de la red y en general de entidades encargadas de la gestión de desastres.

\section{Objetivo General}

Proponer estrategias para la integración de Recursos Energéticos Distribuidos en el nivel de distribución del sistema eléctrico para su participación como oferente de energía y servicios auxiliares ante la ocurrencia de desastres que permitan incrementar la resiliencia del sistema.

\section{Objetivos Específicos}

- Plantear una plataforma de acceso en el espacio de Computación en la nube para el manejo de la información de la red, las transacciones del nivel de distribución que faciliten la integración de DER y su interacción con el Operador de red y entidades que coordinan la gestión de atención de desastres.

- Diseñar estrategias para el mejoramiento de la resiliencia del sistema eléctrico ante desastres naturales apoyados en la utilización de DER.

- Plantear modelos matemáticos para la gestión de DER de manera óptima ante la ocurrencia de un desastre natural, asegurando parámetros mínimos de calidad para la red. 


\section{Metodología}

Dentro de las estrategias metodológicas a utilizar se plantea la revisión de documentación especializada referente a la temática a tratar, que implica una selección, clasificación y análisis de la misma.

Posteriormente, se realizará la modelación matemática del comportamiento de componentes y de las interrelaciones entre los mismos. Las fases que se han planteado en el siguiente proyecto orientado a dar respuesta a los objetivos específicos planteados son las siguientes:

- Una fase transversal que comprende una fundamentación teórica permanente durante el desarrollo de la investigación.

- F1: Plantear una plataforma de acceso en el espacio de Computación en la nube para el manejo de la información de la red, las transacciones del nivel de distribución que faciliten la integración de DER y su interacción con el Operador de red y entidades que coordinan la gestión de atención de desastres.

- F2: Diseñar estrategias para el mejoramiento de la resiliencia del sistema eléctrico ante desastres naturales apoyados en la utilización de DER.

- F3: Plantear modelos matemáticos para la gestión de DER de manera óptima ante la ocurrencia de un desastre natural, asegurando parámetros mínimos de calidad para la red.

- F4: En esta fase se realizará el proceso de verificación y pruebas de los modelos matemáticos y estrategias de integración de DER y mejoramiento de la resiliencia de los sistemas eléctricos ante la ocurrencia de desastres. 


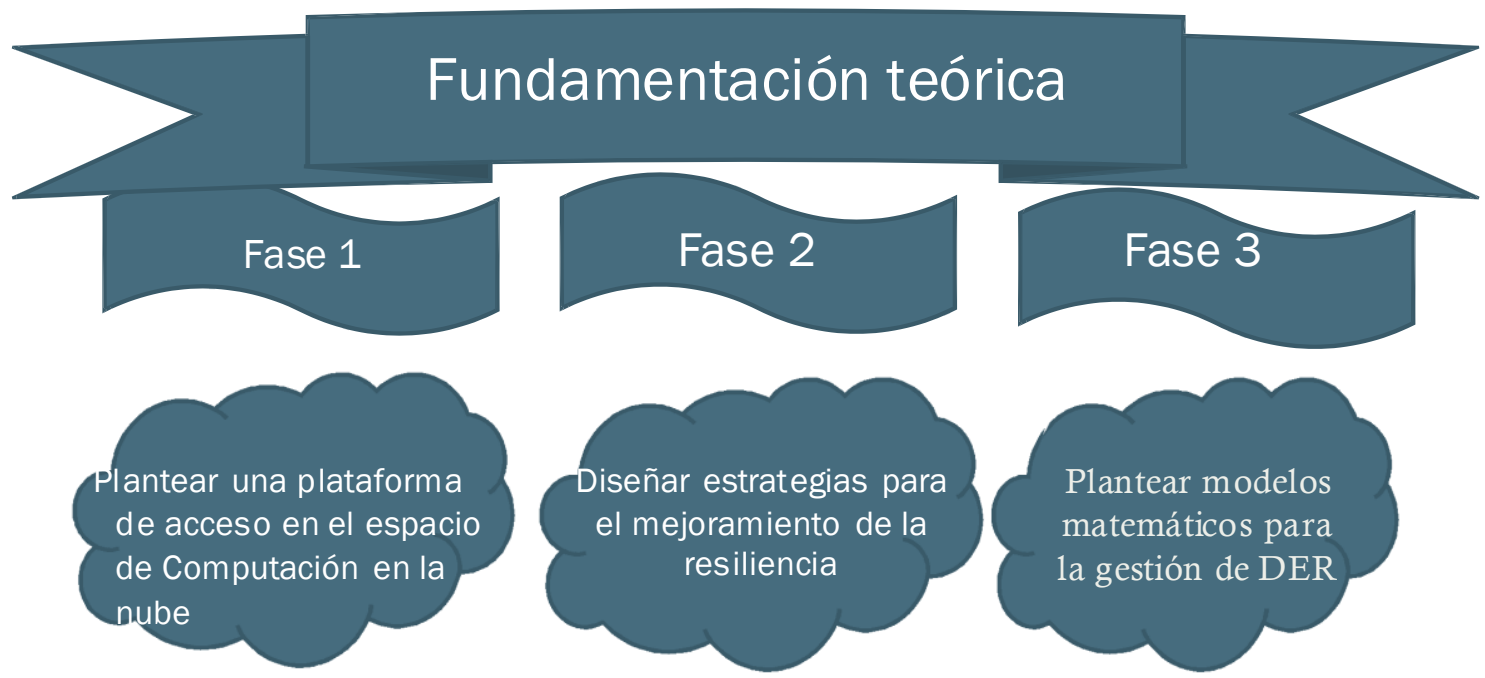

BIOTECH \& ENGINEERING Untels. Jul - Dic.1(2), 2021; ISSN:2788 -4295; 111-129 


\section{Fase 4}

\section{Realizar el proceso de verificación y pruebas de los modelos \\ matemáticos y estrategias de integración de DER}

Figura 4. Fases del trabajo del desarrollo de la investigación

\section{Resultados}

El proyecto de investigación aún se encuentra en curso, no obstante, ya se tiene resultados puntuales como el diseño de una estrategia de seccionamiento de la red ante fallos, tomando como fundamentación matemática la teoría de grafos con la inclusión aleatoria de fuentes de generación distribuida. De igual forma se han realizado pruebas de los perfiles de tensión en la red momentos previos y posteriores a la ocurrencia de un eventual desastre, evaluando el comportamiento en cada los cuales se haya incluido mecanismos de control eficiente de cargas fundamentados en elementos de respuesta de la demanda.

Actualmente se desarrolla una propuesta en MATLAB, que cuenta con una interfaz gráfica para el desarrollo de la actividad de seccionamiento de la red ante fallo. Dicho programa se basa en el modelo de grafos donde los nodos representan los lugares de abastecimiento o producción de energía y usuarios conectados a la red. En caso de un desastre Natural se busca conocer los puntos más cercanos o formas más factibles para el abastecimiento de cargas sensibles del sistema como hospitales, centros de alimentos y atención entre otros con el fin de lograr la resiliencia energética de la red eléctrica afectada. 
La Figura 5, muestra la primera pantalla de la interfaz, en donde todos los espacios están en blanco. La parte superior izquierda es un bloque, Entradas, que permite un máximo de 32 o número de nodos que se van a trabajar, una vez se ingresa el número de nodos, se le da a $S A V E$ y luego BEGIN y en ese instante las letras $I$ y $j$ cambian a números, los cuales corresponden a la conexión entre nodos.

El usuario debe elegir si los nodos que se muestran están o no conectados, una vez seleccione uno, le da a siguiente nodo y muestra la siguiente conexión. Como aún está en desarrollo, se está trabajando en la repetición de los nodos a la hora de elegir, ejemplo si 1 y 2 están conectados, luego se vuelve a preguntar por 2 y 1, que se debe repetir según lo deseado por el usurario; también se está mirando en no mostrar los mismos nodos entre sí para evitar la obviedad, por ejemplo 1 y 1 , o 2 у 2.

El bloque inferior de la derecha, matrices, muestra las diferentes tablas que forman la matriz; en ADYACENCIA se muestra la conexión entre los nodos en donde si están conectados muestra un 1 dentro de la celda correspondiente y si no es así, mostrará un 0; GRADO mostrará la cantidad de veces que cada nodo está conectado; LAPLACIANA muestra la matriz guía que se usa, como su nombre lo dice, con la trasformada de Laplace, y esta es fundamental para que el programa halle el punto que más se vería afectado y a la vez, el seccionamiento que ayudaría a una rápida resiliencia. Por último AUTOVECTORES da la dirección y ubicación del nodo una vez se grafique 


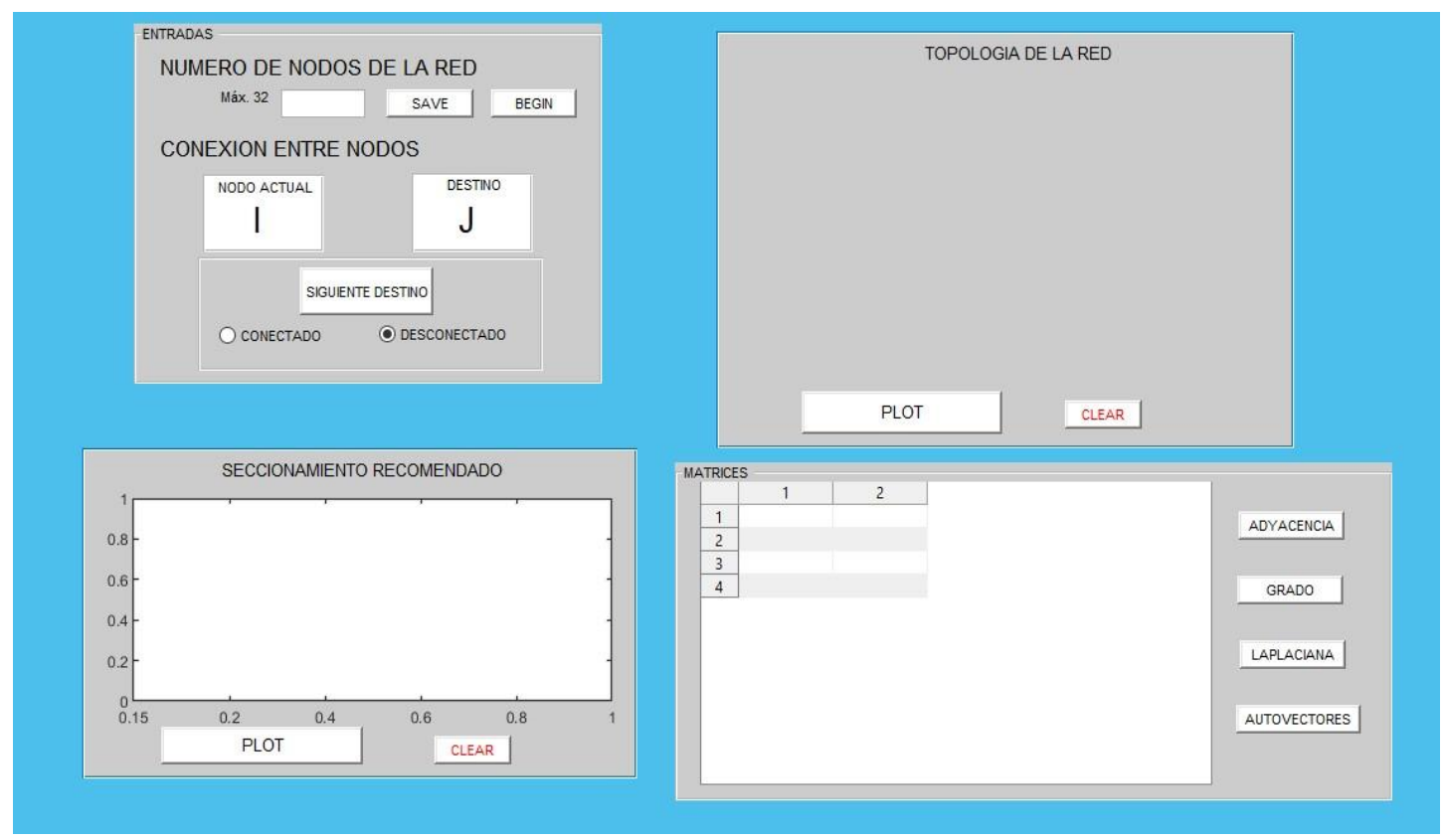

Figura 5. Estructura Inicial - Presentación Matlab

La figura 6, muestra un ejemplo del funcionamiento del programa, en este caso se trabajaron 7 nodos a los cuales se les da las siguientes conexiones:

1-2 sí; 1-3 si, 1-4 no; 1-5 no; 1-6 no; 1-7 no

2-3 sí; 2-4 sí; 2-5 no; 2-6 no; 2-7 no

3-4 sí; 3-5 no; 3-6 no; 3-7 sí

4-5 sí; 4-6 no; 4-7 sí

5-6 sí- 5-7 no 


\section{6-7 sí}

Una vez se mostró la conexión 7-7, y se le da a siguiente nodo, se dejan de mostrar configuraciones, es decir se queda en 7-7, a partir de ese momento se pueden mirar las configuraciones del bloque de matrices seleccionando cualquiera de las deseadas.

La parte inferior izquierda, al darle en PLOT mostrará en el mismo espacio el seccionamiento recomendado, es decir, el punto la línea trasversal representa un corte en la red, por lo que los puntos superiores, se pueden conectar entre sí para mantenerse en operación, y de igual manera pasa con los puntos inferioes.

La parte superior derecha muestra la topología de la red o mapeo de nodos, en donde muestra la conexión entre los diferentes nodos, la cual coincide con la información digitada en el bloque ENTRADAS. Como otras operaciones del programa, aún se está trabajando en una topología de red más clara y con menos cruces entre sí.

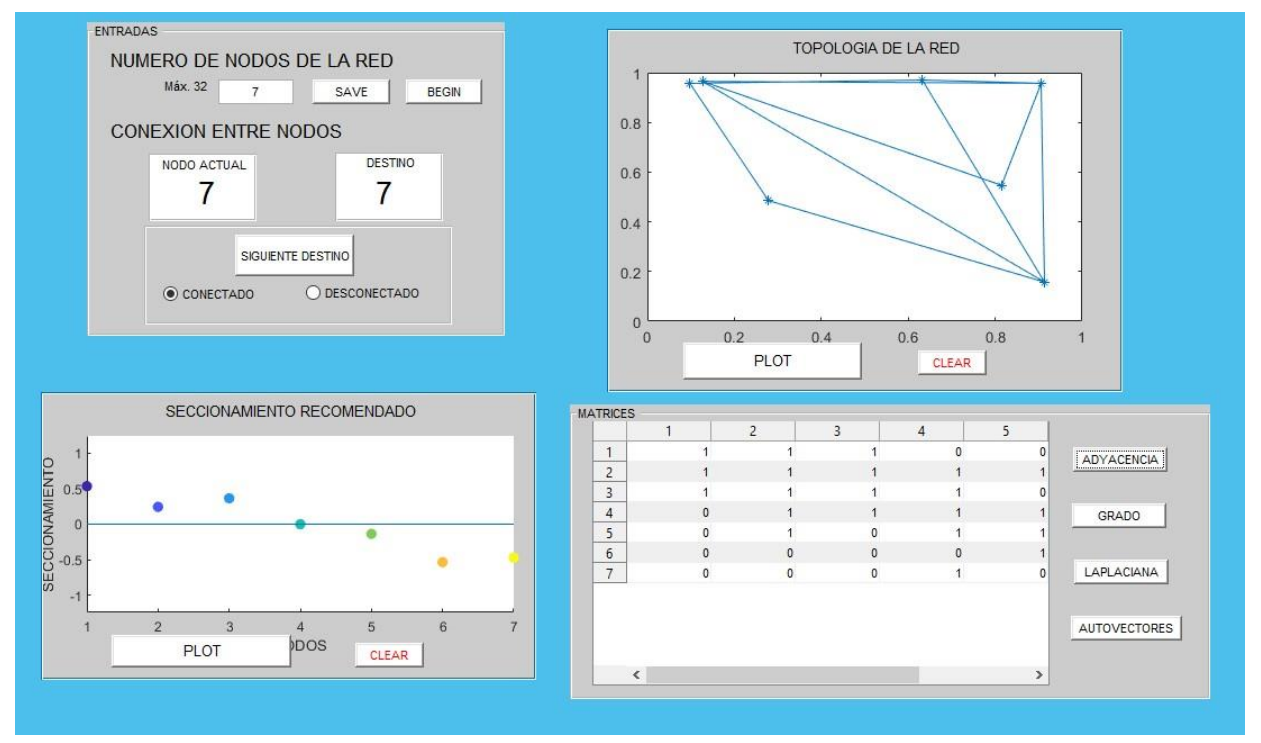

Figura 6. Estructura Resultado Parcial - Tipología de la Red

BIOTECH \& ENGINEERING Untels. Jul - Dic.1(2), 2021; ISSN:2788 -4295; 111-129 


\section{Conclusiones}

El documento presentado sintetiza aspectos globales de la investigación, presentando que se logró, el significado de resultados y trabajos futuros.

Como se puede ver la tendencia en el desarrollo de las investigaciones sobre resiliencia y su integración con recursos energéticos distribuidos muestra que el sistema de gestión redes ante desastres está tomando importancia demostrando la preocupación de una buena gestión por lo que además complementándose a los recursos energéticos distribuidos muestran el camino a seguir en tema de resiliencia a través de los años (2024)

Con el desarrollo de la aplicación en MATLAB se logra el seccionamiento de la red, pero aun vemos que existen problemas en las líneas de los nodos ya que se cruzan entre ellas, pero se está trabajando.

\section{Bibliografía}

Abeysinghe, S., Wu, J., Sooriyabandara, M., Abeysekera, M., Xu, T., \& Wang, C. (2018).

Topological properties of medium voltage electricity distribution networks. Applied Energy, 210, 1101-1112. https://doi.org/10.1016/j.apenergy.2017.06.113

Albert, R., Albert, I., \& Nakarado, G. L. (2004). Structural vulnerability of the North American power grid. Physical Review E - Statistical, Nonlinear, and Soft Matter Physics, 69(2 2), 1-10. https://doi.org/10.1103/PhysRevE.69.025103

Andersson, G., Donalek, P., Farmer, R., Hatziargyriou, N., Kamwa, I., Kundur, P, Vittal, V. 
(2005). Causes of the 2003 major grid blackouts in North America Europe, and recommended means to improve system dynamic performance. IEEE Transactions on Power Systems, 20(4), 1922-1928. https://doi.org/10.1109/TPWRS.2005.857942

Bajwa, A. A., Mokhlis, H., Mekhilef, S., \& Mubin, M. (2019). Enhancing power system resilience leveraging microgrids: A review. Journal of Renewable and Sustainable Energy, 11(3), 035503. https://doi.org/10.1063/1.5066264

Bouffard, F., \& Kirschen, D. S. (2008). Centralised and distributed electricity systems. Energy Policy, 36(12), 4504-4508. https://doi.org/10.1016/j.enpol.2008.09.060

Bryant, V., \& Wallis, W. D. (2001). A Beginner's Guide to Graph Theory. The Mathematical Gazette, 85(503), 374. https://doi.org/10.2307/3622074

CREG (Comisión de Regulación de Energía y Gas). (2010). Comisión de Regulación de Energía y Gas. 1-36. http://www.creg.gov.co/index.php/es/creg/quienes-somos/historia

Di Somma, M., Yan, B., Bianco, N., Graditi, G., Luh, P. B., Mongibello, L., \& Naso, V. (2015). Operation optimization of a distributed energy system considering energy costs and exergy efficiency. Energy Conversion and Management, 103, 739-751. https://doi.org/10.1016/j.enconman.2015.07.009

Hammons, T. J. (2008). Integrating renewable energy sources into European grids. International Journal of Electrical Power \& Energy Systems, 30(8), 462-475. https://doi.org/10.1016/j.ijepes.2008.04.010

International Energy Agency. (2013). World Energy Outlook 2013. Agencia Internacional de Energía, 7. https://doi.org/10.1787/weo-2013-en

Kassakian, J. G., Schmalensee, R., Desgroseilliers, G., Heidel, T. D., Afridi, K., Farid, a M., Grochow, J. M., Hogan, W. W., Jacoby, H. D., Kirtley, J. L., \& Others. (2011). The future of the electric grid. In Massachusetts Institute of Technology, Tech. Rep. http://mitei.mit.edu/publications/reports-studies/future-electric-grid Landau, L. (Universidad A. D. O. (1937). Calidad de la energia electrica. Zhurnal Eksperimental'noi i Teoreticheskoi Fiziki. http://scholar.google.com/scholar?hl=en\&btnG=Search\&q=intitle:No+Title\#0 
Levin, T., \& Thomas, V. M. (2016). Can developing countries leapfrog the centralized electrification paradigm? Energy for Sustainable Development, 31, 97-107. https://doi.org/10.1016/j.esd.2015.12.005

Niknam, T., Ranjbar A.M., Shirani A.R., and O. A. (2005). C i r e d 18. June, 6-9.

Panteli, M., \& Mancarella, P. (2015). The Grid: Stronger, Bigger, Smarter? IEEE Power \& Energy Magazine, May/June, 58-66. https://doi.org/10.1109/MPE.2015.2397334

Quintero, S. C. (2013). Ancillary Services Review in Electrical Power Systems in desregulated Market.

Sadeghi, A., Larimian, T., \& Molabashi, A. (2012). Evaluation of Renewable Energy Sources for Generating Electricity in Province of Yazd: A Fuzzy Mcdm Approach. Procedia - Social and Behavioral Sciences, 62, 1095-1099. https://doi.org/10.1016/j.sbspro.2012.09.187

Sismotto, F., \& Hage, E. L. (2005). An evolutionary algorithm for the optimisation of distributed generation dispatch. 18th International Conference on Electricity Distribution. Turin, Italy. June 6-9 2005, June, 6-9. 